\section{MS3-P6 Novel data collection and processing strategies for the next generation X-ray source MAX IV}

Jie Nan ${ }^{1}$, Mikel Eguiraun ${ }^{1}$, Fredrik Bolmsten ${ }^{1}$, Antonio Milan Ote $^{1}$, Alberto Nardella ${ }^{1}$, Thomas Ursby ${ }^{1}$, Johan Unge $^{1}$, Marjolein MGM Thunnissen ${ }^{1}$ \& U Uwe Mueller

1. MAX IV Laboratory

email: jie.nan@maxiv.lu.se

MAX IV is the first 'fourth-generation' synchrotron in the world [1] and it will start to produce the brightest light from this summer and onwards. As the first MX beamline at MAX IV, BioMAX will not only have highly brilliant X-ray beam but also high performance hardware $[2,3]$, including the next generation hybrid pixel-array detector EIGER $16 \mathrm{M}$, a newly developed fast sample changer ISARA and a high precision MD3 diffractometer.

To take full advantage of these cutting edge technologies and to provide a more user friendly software interface particularly for the arising remote access, a new web-based version of a beamline and experiment control software MXCuBE3 has been being developed by MAX IV in strong collaboration with the ESRF. The software will support both single sample and in situ plate manipulation with modern acquisition methods, such as mesh scan and raster scan. Users can carry out their experiments through web browsers from any place and any operating system, without installation of any additional software.

Following these high throughput experiments, a fast and accurate data processing pipeline becomes critical to meet the high data rate. While testing and comparing various existing processing pipelines that utilize XDS and Dials, we are also seeking new approaches that can handle the diffraction images. Finally, the experimental meta-data and processing results will be stored in our ISPyB server and users can access them via web browsers.

[1] D, Castelvecchi. (2015) Nature.

[2] M.M.G.M. Thunnissen et al. (2013) J. Phys. Conf. Ser.

[3] M.M.G.M. Thunnissen et al. (2015) Acta Cryst. A

Keywords: BioMAX, MAX IV, data collection, data processing

\section{MS3-P7 Pushing the Limits of a Beamline Endstation}

Darren A. Sherrell

\section{Diamond Light Source}

email: darren.sherrell@diamond.ac.uk

The recently upgraded endstation at beamline I24, Diamond Light Source greatly facilitates novel modes of data collection. The endstation can accommodate in situ 96 well crystal plates using a horizontal goniometer, conventional cryo-cooled rotation kappa data collection on a low sphere of confusion vertical goniometer, and the high speed serial delivery of crystals on fixed targets such as a silicon chip. The small beamsize $(\sim 2 \times 5 \mu \mathrm{m} 2)$ and high flux $(>1 \times 10-12 \mathrm{ph} \mathrm{s}-1)$ available at the beamline has necessitated the development of new approaches to motion control, data collection and data analysis for the full potential and flexibility of the new endstation to be realised.

The difficulty in aligning micro-crystals on pins, in situ and in fixed targets in the X-ray beam and then the short duration of the diffraction experiment raises different and interesting technological challenges. The new endstation at 24 couples state-of-the-art commercially available hardware, with bespoke motion programs and novel software interfaces to allow high speed, high precision novel experiments. I will describe I24's versatile endstation design, and the advances in each of many techniques that have been achieved by our group. The techniques couple traditional techniques with serial approaches at both synchrotron and XFEL sources.

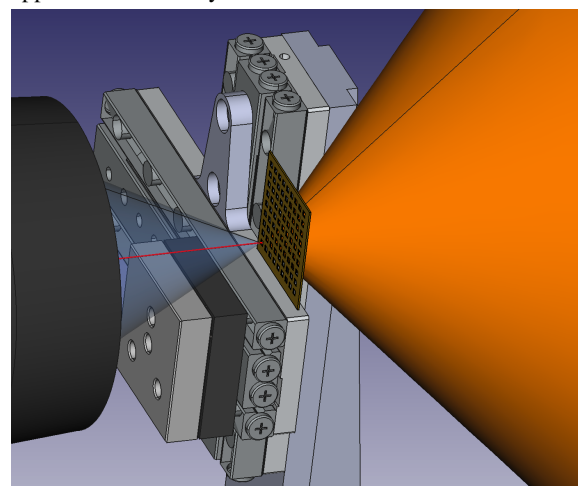

Figure 1. CAD drawing of a fixed target array with $2 \AA$ diffraction cone and focus cone of the on-axis objective lens.

Keywords: In situ, serial crystallography, microfocus, sample delivery, XFEL 